\title{
Mutant KRAS drives metabolic reprogramming and autophagic flux in premalignant pancreatic cells
}

\author{
Tatsunori Suzuki $^{1} \cdot$ Takahiro Kishikawa $^{1} \cdot$ Tatsuyuki Sato $^{2} \cdot$ Norihiko Takeda $^{2} \cdot$ Yuki Sugiura $^{3} \cdot$ Takahiro Seimiya $^{1} \cdot$

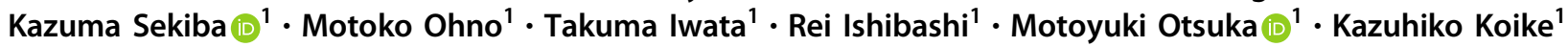

Received: 31 July 2020 / Revised: 22 February 2021 / Accepted: 23 March 2021 / Published online: 8 April 2021

(c) The Author(s) 2021. This article is published with open access

\begin{abstract}
Mutational activation of the KRAS gene occurs in almost all pancreatic ductal adenocarcinoma (PDAC) and is the earliest molecular event in their carcinogenesis. Evidence has accumulated of the metabolic reprogramming in PDAC, such as amino acid homeostasis and autophagic flux. However, the biological effects of KRAS mutation on metabolic reprogramming at the earlier stages of PDAC carcinogenesis are unclear. Here we report dynamic metabolic reprogramming in immortalized human non-cancerous pancreatic ductal epithelial cells, in which a KRAS mutation was induced by gene-editing, which may mimic early pancreatic carcinogenesis. Similar to the cases of PDAC, KRAS gene mutation increased the dependency on glucose and glutamine for maintaining the intracellular redox balance. In addition, the intracellular levels of amino acids were significantly decreased because of active protein synthesis, and the cells required greater autophagic flux to maintain their viability. The lysosomal inhibitor chloroquine significantly inhibited cell proliferation. Therefore, metabolic reprogramming is an early event in carcinogenesis initiated by KRAS gene mutation, suggesting a rationale for the development of nutritional interventions that suppress or delay the development of PDAC.
\end{abstract}

\section{Introduction}

Pancreatic ductal adenocarcinoma (PDAC) is the fourth leading cause of cancer-related death in the United States [1]. The 5-year survival rate of patients with PDAC is $9 \%$ [1] and it is predicted that PDAC will, in the near future, become the second leading cause of cancer-related death [2]. The development of interventions that interrupt carcinogenesis will require an understanding of the early biological events during PDAC carcinogenesis.

Supplementary information The online version contains supplementary material available at https://doi.org/10.1038/s41417021-00326-4.

Motoyuki Otsuka

otsukamoto@g.ecc.u-tokyo.ac.jp

1 Department of Gastroenterology, Graduate School of Medicine, The University of Tokyo, Tokyo 113-8655, Japan

2 Department of Cardiovascular Medicine, Graduate School of Medicine, The University of Tokyo, Tokyo 113-8655, Japan

3 Department of Biochemistry, Keio University School of Medicine, Tokyo 160-8582, Japan
The acquisition of a gain-of-function mutation in KRAS is the predominant driver of malignant transformation in various tissues [3]. Notably, more than $90 \%$ of PDAC cases have KRAS mutations [4-8], and approximately $50 \%$ of colorectal cancer has mutations in $R A S$ or a $R A S$-related pathway [9]. The development of PDAC is characterized by a series of gene mutations, and the acquisition of the KRAS mutation is the earliest change in pancreatic carcinogenesis [10-12].

KRAS functions as a binary switch that cycles between an active GTP-bound and inactive GDP-bound state [13-16]. Mutant KRAS activates a diverse spectrum of downstream effector proteins [17], particularly components of the mitogen-activated protein kinase (MAPK) pathway and of the phosphoinositide-3-kinase (PI3K) pathway. KRAS mutation in PDAC is critical for reprogramming intracellular metabolism of, for example, glucose and glutamine, to facilitate the rapid proliferation of cancer cells [18-27]. To acquire sufficient nutrients for rapid proliferation, PDAC has elevated basal autophagy [28-30] in which biomolecules digested in lysosomes become available as nutrients [31, 32].

Although KRAS gene mutation is related to metabolic reprogramming in cancer cells $[20,26]$ or in genetically engineered mouse models [33,34], it's biological effects on 
metabolic reprogramming in human cells as early events in PDAC carcinogenesis are unclear. We report here dynamic metabolic reprogramming in immortalized human pancreatic duct epithelial cells with KRAS mutation $\left(K R A S^{\mathrm{G} 12 \mathrm{~V}}\right)$ induced by gene-editing, which may mimic early pancreatic carcinogenesis. Although most studies of the KRAS oncogene involved overexpression of a mutant $K R A S$ gene, these models do not always recapitulate early events of carcinogenesis because excessive expression of mutant KRAS driven by a constitutive promoter may affect its physiological function. The tissue of origin also determines whether transforming mutations initiate carcinogenesis [35]. Thus, we compared the phenotypes of heterozygous mutant $K R A S$ genetically knocked-in to pancreatic epithelial cells and mutant KRAS gene-overexpressing cells, and mutant KRAS gene-overexpressing human colon epithelial cells.

\section{Results}

\section{Establishment of human non-cancerous epithelial cells with KRAS mutation}

To investigate the biological effects of KRAS mutation in human pancreatic non-cancerous epithelial cells, we introduced using the clustered regularly interspaced short palindromic repeats (CRISPR)/Cas9 system in a G12V mutation in the KRAS gene in HPNE cells, an immortalized human pancreatic primary epithelial cell line. According to a previous report [36], to isolate cells with $K R A S$ mutation repeated limiting dilution followed by assessment of mutation frequencies by droplet-digital PCR (ddPCR) was carried out (Fig. 1a). To eliminate the influence of the remaining ssODN, ddPCR was performed after nested PCR using primer pairs set outside the ssODN sequences. After four selection cycles, two clones, \#152622 and \#152623 were isolated, in which the KRAS gene mutation frequencies were $50 \%$, indicating the successful introduction of heterozygous KRAS mutation (Supplementary Fig. 1a-d). Hereafter, we used clones \#152622 (HPNE-cKRAS cells) and \#152623 (HPNE-cKRAS2 cells). Lentivirus-mediated HA-tagged mutant KRAS (G12V) gene-overexpressing polyclonal HPNE cells (HPNE-vKRAS) and overexpressing immortalized non-cancerous human colon primary epithelial cells (colon-vKRAS), were used as references. To investigate the difference between HPNEcKRAS and HPNE-vKRAS cells, we examined the mutant KRAS copy number in HPNE-vKRAS cells by subcloning several single clones. The KRAS gene mutation frequencies were unexpectedly nearly $33 \%$ in all isolated HPNEvKRAS clones, indicating that only one copy of mutant KRAS was inserted into the genome, with two wildtype alleles (Supplementary Fig. 1e). However, mutant KRAS expression varied among clones; therefore, we examined the HPNE-vKRAS2 clone (hereafter HPNE-vKRAS ${ }_{\text {high }}$ cells) that expressed the highest amount of mutant KRAS in several experiments to examine whether biological functions were affected by the expression of mutant KRAS (Supplementary Fig. 1f).

Mutant KRAS expression levels in HPNE-cKRAS, HPNE-vKRAS, and colon-vKRAS cells were determined by western blotting using a KRAS G12V mutant-specific antibody (Fig. 1b and Supplementary Fig. 2a). Consistent with a previous report [37], phosphorylation of ERK and AKT was modestly induced in HPNE-cKRAS and HPNEvKRAS cells (Fig. 1b). In contrast, the PI3K-AKT pathway was significantly activated, and ERK phosphorylation was modestly induced, in colon-vKRAS cells (Supplementary Fig. 2a). The RAS activities were almost in accordance with the levels of mutant KRAS protein expression (Fig. 1c and Supplementary Fig. 2b). The growth rate of HPNE-cKRAS and HPNE-vKRAS cells was increased (Fig. 1d), but that of colon-vKRAS cells was not (Supplementary Fig. 2c). Therefore, while KRAS mutation induces cell survival and intracellular signaling, the biological effects of KRAS mutation vary among tissues.

\section{The growth of KRAS mutant cells is dependent on glucose and glutamine}

KRAS mutations alter glucose and glutamine metabolism in cancerous cells $[18,26]$. To evaluate the metabolic changes induced by KRAS mutation in non-cancerous cells, we examined the dependence of glucose and/or glutamine on the growth of human non-cancerous pancreatic and colon epithelial cells with $K R A S$ mutations. Compared to KRAS wildtype cells, the proliferation of HPNE-cKRAS and HPNEvKRAS cells was significantly suppressed in glucosedeficient and/or glutamine-deficient medium (Fig. 2a). In colon epithelial cells, colon-vKRAS cells showed similar tendencies, although not significant regarding glutamine (Supplementary Fig. 3a). These results suggest that the growth of cells with KRAS mutation depends more on glucose or glutamine. Consistently, the expression levels of glucose transporter (GLUT1), rate-limiting glycolytic enzyme (HK2), and enzymes enhancing aerobic glycolysis (LDHA and PDK1) (Fig. 2b and Supplementary Fig. 3b-d), as well as glucose uptake (Fig. 2c and Supplementary Fig. 3e) were increased in HPNE-cKRAS, HPNE-vKRAS, and colonvKRAS cells. In addition, the lactate level, which reflects enhanced glycolytic flux, was increased in HPNE-cKRAS and HPNE-vKRAS cells (Fig. 2d), whereas it was not significantly changed in colon-vKRAS cells (Supplementary Fig. 3f). Moreover, the expression levels of glutamine metabolism-related enzymes such as glutaminase 1 (GLS1), glutamate dehydrogenase 1 (GLUD1), and transaminases 
a

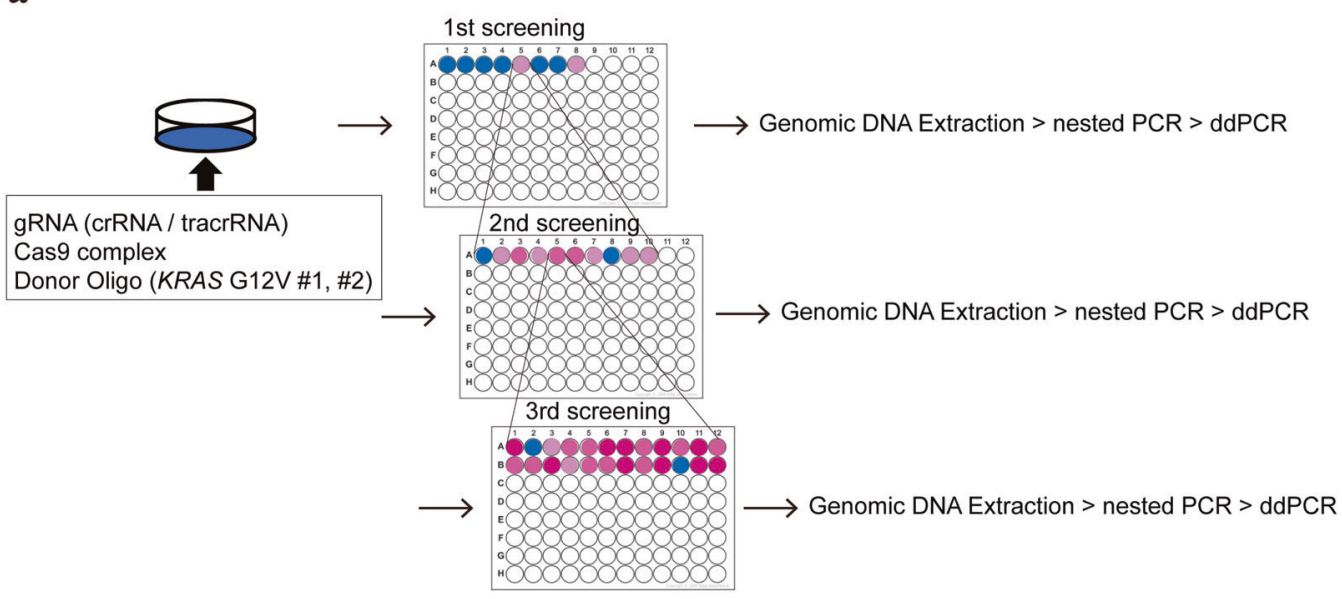

b

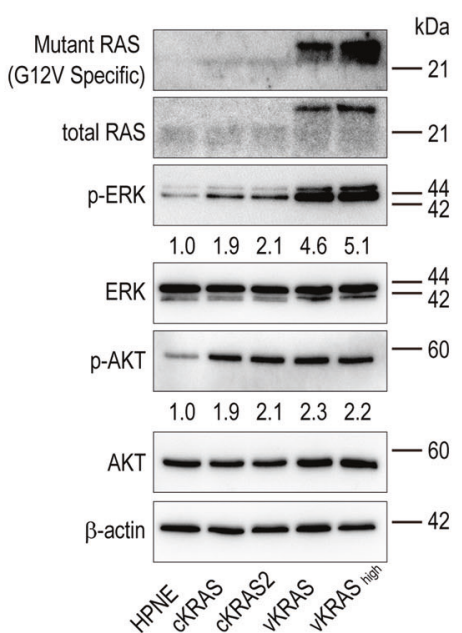

d

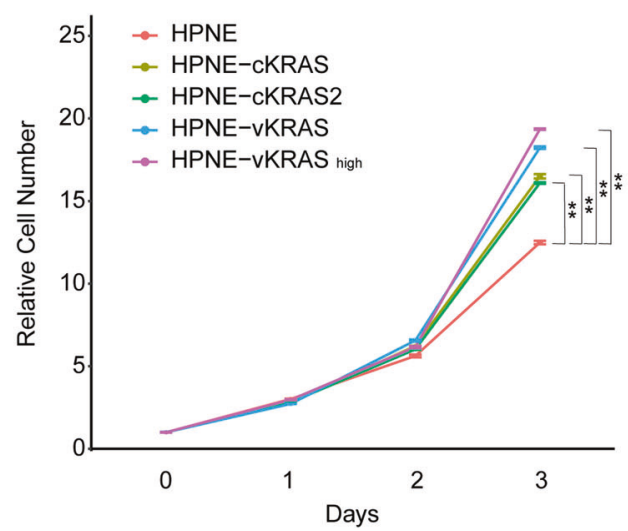

Fig. 1 Establishment of gene-edited KRAS-mutant pancreatic epithelial cells. a Schema of cell selection. Gene-edited cells generated by transient transfection with gRNA, Cas9 complex, and ssODN, were sequentially selected by limiting dilution and ddPCR, yielding geneedited monoclonal cells, blue and red wells, wild-type and KRAS mutant cells, respectively. b Determination of the levels of mutant KRAS $(\mathrm{G} 12 \mathrm{~V})$ (genome-edited in cKRAS and HA-tagged mutant KRAS expression in vKRAS) and of related intracellular signaling molecules by Western blotting. The vKRAS construct has an HA-tag

\section{c}

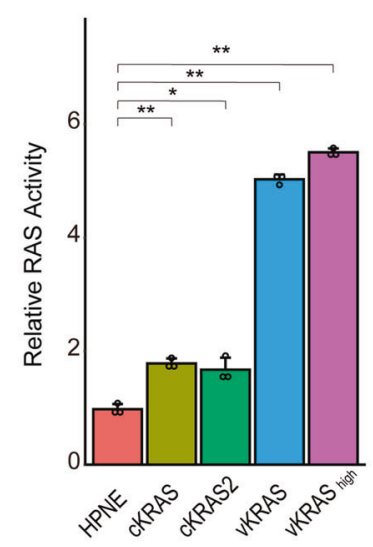

and is a slightly heavier molecule. Representative results from three independent experiments are shown. Band intensities of phosphorylated ERK (p-ERK) and phosphorylated AKT (p-AKT) are indicated below the images. $\mathbf{c}$ Determination of RAS activity by ELISA. Results are the average of three biological replicates; error bars represent the SD. $* p<0.05 ; * * p<0.01$. d Cell growth curve. Cell numbers relative to those at day 0 are indicated. Results are the average of three biological replicates; error bars represent SD. $* * p<0.01$. 
a
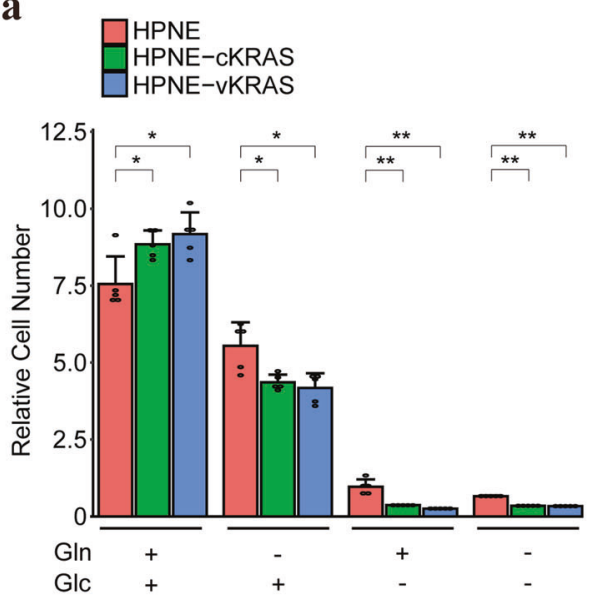

c

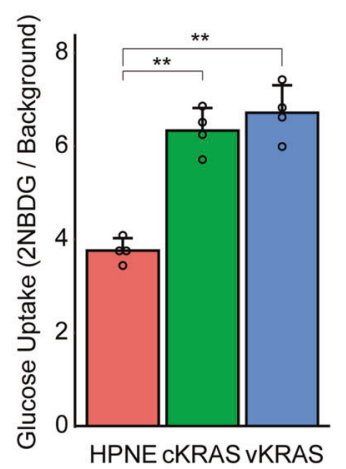

e
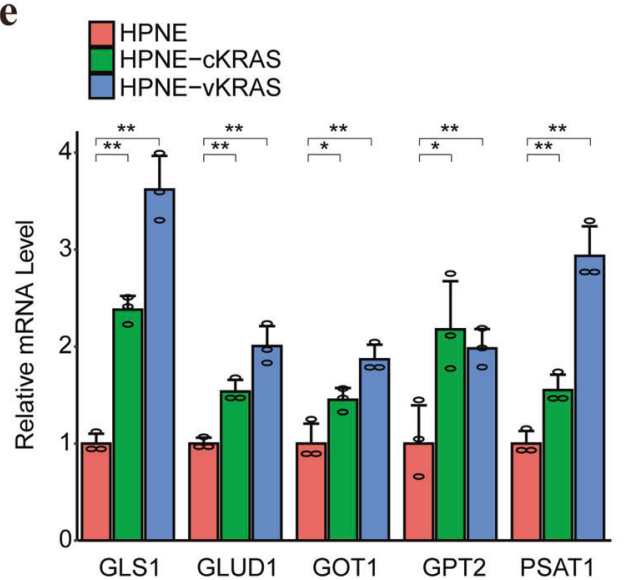

Fig. 2 KRAS mutant pancreatic epithelial cells are dependent on glucose and glutamine. a Cell growth rate in the presence or absence of glucose (Glc) and glutamine (Gln). The relative cell number was determined by measuring cell numbers at days 0 and 3 . Results are the means of five biological replicates. Error bars represent the SD. $* p<$ $0.05 ; * * p<0.01$. b Relative mRNA levels of the indicated genes. The levels in control HPNE cells were set as 1 . Results are the means of three biological replicates; error bars represent the SD. $* p<0.05 ; * * p$ b
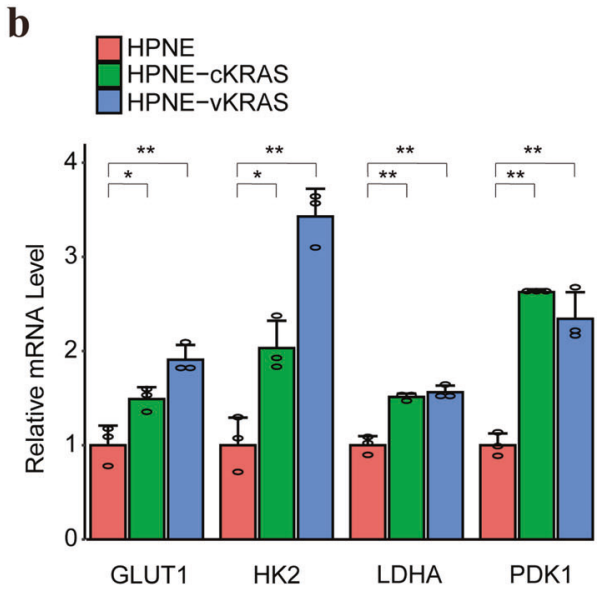

d

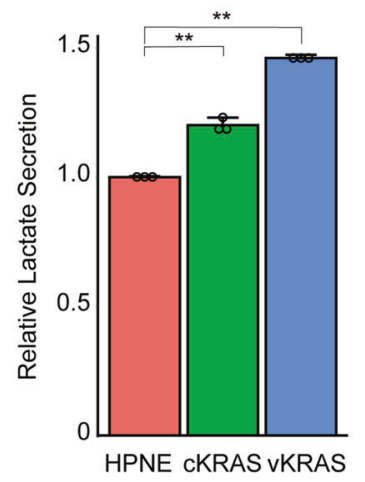

$<0.01$. c Glucose uptake levels were measured using 2-NBDG. Results are the average of four independent replicates. Error bars represent SD. ${ }^{* *} p<0.01$. d Lactate secretion levels are shown. Results are the average of three independent replicates. Error bars represent SD. ${ }^{*} p<0.01$. e Relative mRNA levels of indicated genes are shown after setting the levels in HPNE cells as 1 . Results are the average of three biological replicates. Error bars represent mean \pm SD. $* p<0.05$; $* * p<0.01$. 
(GOT1, GPT2, and PSAT1) were increased (Fig. 2e and Supplementary Fig. 3c, d, and g), suggesting that glutamine utilization was increased in HPNE-cKRAS, HPNE-vKRAS, and colon-vKRAS cells. These different expression profiles of metabolic genes in mutant KRAS cells may be linked to the mutant KRAS expression and RAS activity levels (Fig. 1b and $\mathrm{c})$.

Therefore, KRAS mutations modulate glucose and glutamine metabolism, which is required for the proliferation of non-cancerous cells with KRAS mutation.

\section{Glutamine is crucial for energy production and maintaining the redox balance in KRAS mutant cells}

Glutamine is converted to $\alpha$-ketoglutarate $(\alpha-K G)$, a substrate for the TCA cycle and energy production, nucleic acid synthesis, and amino acid synthesis [38]. Because mutant KRAS enhanced glutamine utilization, we hypothesized that the flow to the TCA cycle increased in non-cancerous cells with $K R A S$ mutation, promoting mitochondrial oxidative phosphorylation, as in murine renal cells expressing oncogenic H-Ras [39]. As expected, the oxygen consumption rate (OCR), which reflects the level of mitochondrial oxidative phosphorylation (OXPHOS), was significantly increased in HPNE-cKRAS and HPNE-vKRAS cells (Fig. 3a). The mitochondrial DNA copy number, which is correlated with the expression levels of mitochondrial metabolic genes [40], was also significantly increased in HPNE-cKRAS and HPNE-vKRAS cells (Supplementary Fig. 4a). Subsequently, the ATP levels were increased in HPNE-cKRAS and HPNEvKRAS cells, which reflects the enhanced glycolysis and OXPHOS (Fig. 3b). To examine the role of glutamine in energy production, $K R A S$ mutant cells were cultured in a glutamine-deficient medium. The ATP levels were significantly decreased to a greater extent in HPNE-cKRAS and HPNE-vKRAS cells than in wild-type cells, compared with those cultured in glutamine-containing medium (Fig. 3c). Similarly, the ATP levels were significantly decreased in $K R A S$ mutant cells following treatment with the glycolysis inhibitor 2-deoxy-D-glucose (2DG) and the OXPHOS inhibitor antimycin, which suggests that both the glycolysis and OXPHOS pathways are dependently utilized to maintain energy resources in the cells with the KRAS mutation (Supplementary Fig. 4b and c).

Intriguingly, although mitochondrial oxidative phosphorylation leads to the production of reactive oxygen species (ROS) [41], the ROS levels were decreased in HPNE-cKRAS and HPNE-vKRAS cells (Fig. 3d), suggesting that these cells maintained the redox balance despite enhanced mitochondrial metabolism. Consistently, the reduced glutathione (GSH)/oxidized glutathione (GSSG) ratio, an index of cellular redox metabolism [42], was maintained in those cells (Fig. 3e).
Glutamine is a substrate for the synthesis of glutathione, a tripeptide (composed of glutamate, cysteine, and glycine) that protects cells from free radical damage by acting as an antioxidant [38]. In PDAC with the KRAS mutation, glutamine metabolism is reprogramed to increase the NADPH/ $\mathrm{NADP}^{+}$ratio through the reactions of transaminases, which can help maintain the cellular redox state [26]. Therefore, based on the hypothesis that mutant KRAS increases glutathione synthesis via glutamine consumption to counter ROS production, KRAS mutant cells were cultured in a glutamine-deficient medium. The ROS levels were significantly increased to a greater extent in HPNE-cKRAS and HPNE-vKRAS cells than in wild-type cells, compared with those cultured in glutamine-containing medium (Fig. 3f). Consistently, the ROS levels were significantly increased in HPNE-cKRAS and HPNE-vKRAS cells compared with wild-type cells following treatment with the transaminase inhibitor aminooxyacetate (AOA) (Supplementary Fig. 4d). These findings suggest that non-cancerous pancreatic cells with KRAS mutation use glutamine for energy production, as well as maintaining the redox balance.

\section{Intracellular amino acid levels are decreased in KRAS mutant pancreatic epithelial cells}

Because metabolic changes were observed in noncancerous cells with KRAS mutation, we next performed comprehensive metabolome analyses of HPNE control, HPNE-cKRAS, and HPNE-vKRAS cells under normal culture conditions. Among the 116 metabolites examined, we focused on the amino acid levels. This was because, although almost all amino acids were reduced in HPNE-cKRAS and HPNE-vKRAS cells, non-essential amino acid levels, particularly asparagine and proline, were significantly reduced (Fig. 4a and b). Although the intracellular asparagine level was significantly reduced in HPNE-cKRAS and HPNE-vKRAS cells, the expression level of asparagine synthetase (ASNS) was not changed (Fig. 4c), suggesting that the reduction in the intracellular asparagine level was not a result of reduction of asparagine synthesis. The expression levels of most amino acid transporters were upregulated in HPNE-cKRAS, HPNEvKRAS, and colon-vKRAS cells (Fig. 4c and Supplementary Fig. 5a). Based on these results, we speculate that amino acids, including asparagine, are transported from extracellular spaces and consumed in cells with KRAS mutation.

To evaluate the amino acid consumption and protein translation levels, we performed a puromycin labeling assay [43]. In a normal medium, protein synthesis was significantly enhanced in HPNE-cKRAS, HPNE-vKRAS, and colon-vKRAS cells (Fig. 4d and Supplementary Fig. 5b), particularly in HPNE-cKRAS cells (Fig. 4d). 
a

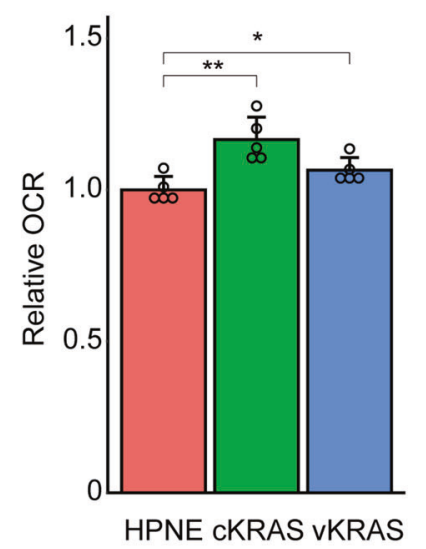

d

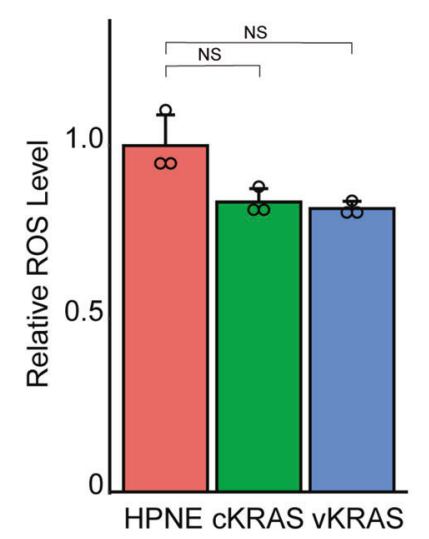

b

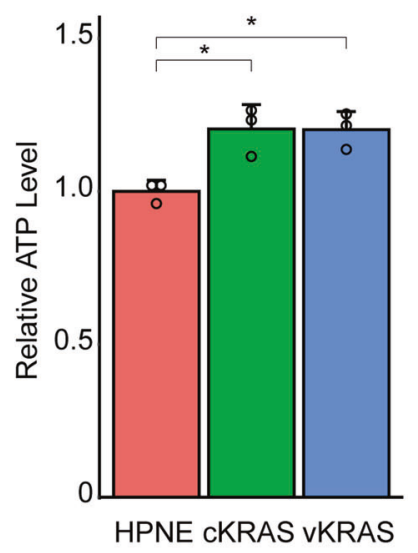

$\mathbf{e}$

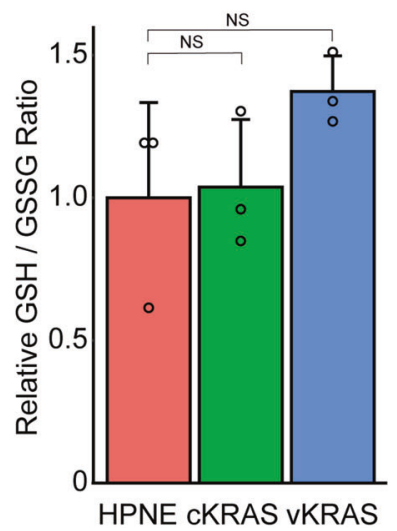

c

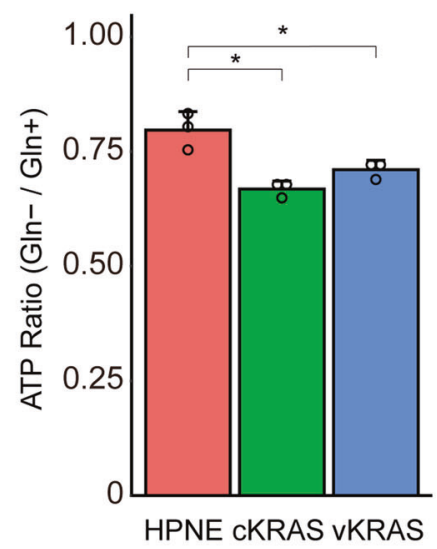

f

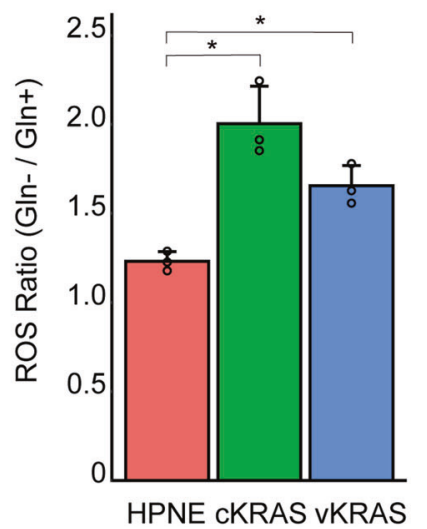

Fig. 3 Glutamine is crucial for energy production and the redox balance in KRAS mutant pancreatic epithelial cells. a Oxygen consumption rate. Results are the means of five biological replicates; error bars represent the SD. $* p<0.05 ; * * p<0.01$. b Intracellular ATP levels. Results are the means of three biological replicates; error bars represent the SD. ${ }^{*} p<0.05$. c Intracellular ATP levels under normal and Gln-deficient conditions with the calculated ratios (ATP levels in Gln-/levels in Gln + ). Results are the means of three biological replicates; error bars represent the SD. ${ }^{*} p<0.05$. d Intracellular ROS

However, in a glutamine-deficient medium, protein synthesis was significantly and similarly suppressed irrespective of the KRAS mutation status (Fig. 4d and Supplementary Fig. 5b), probably because glutamine is required for uptake of essential amino acids [43, 44]. Consistently, mTOR activity, as indicated by the phosphorylated p70S6K and phosphorylated 4E-BP1 levels, was enhanced in HPNE-cKRAS and HPNE-vKRAS cells independent of AMPK status (Fig. 4e). Moreover, we observed that the mTOR inhibitor rapamycin suppressed protein synthesis (Fig. 4f). Collectively, these data suggest that the mTOR pathway is more activated and biomass synthesis is enhanced in cells with KRAS mutation, levels. Results are the means of three biological replicates; error bars represent the SD. NS, not significant. e GSH/GSSG ratio assessed from the metabolome data and relative ratios taking the value of control HPNE cells as 1. Results are the means of three biological replicates; error bars represent the SD. NS, not significant. f Intracellular ROS levels under normal and Gln-deficient conditions with the calculated ratios (ROS levels in Gln-/levels in Gln+). Results are the means of three biological replicates; error bars represent the SD. $* p<$ 0.05 .

which leads to significant depletion of the intracellular amino acid levels.

\section{Autophagy is required for the maintenance of KRAS mutant cells}

Amino acids, such as asparagine and glutamine, are essential for cell growth [43-45]. Because amino acid consumption was enhanced in HPNE-cKRAS and HPNEvKRAS cells, rendering them nutrient-deficient, we hypothesized that autophagy is upregulated to promote amino acid-recycling. As expected, the autophagic flux was increased in HPNE-cKRAS, HPNE-vKRAS, and 
a

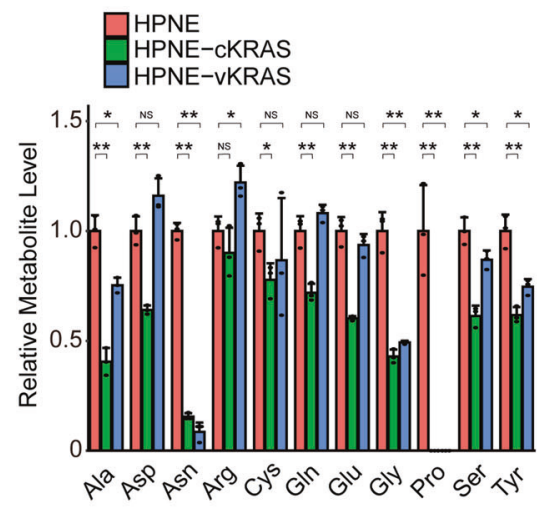

c

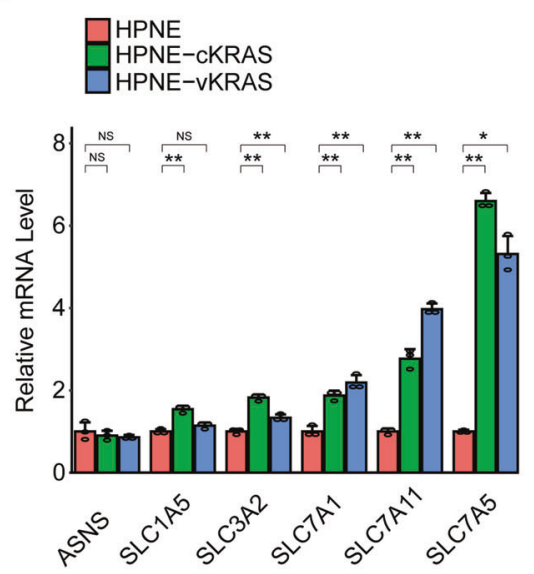

e

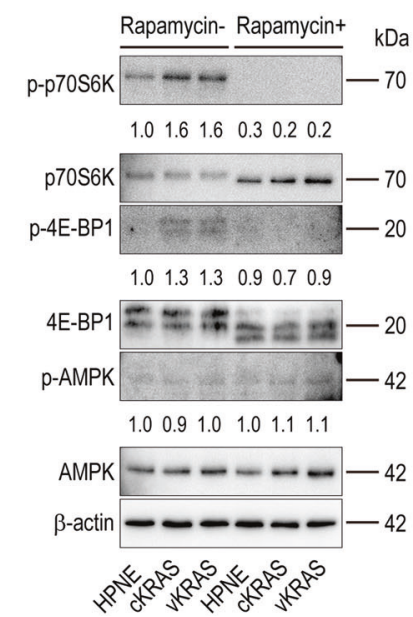

b

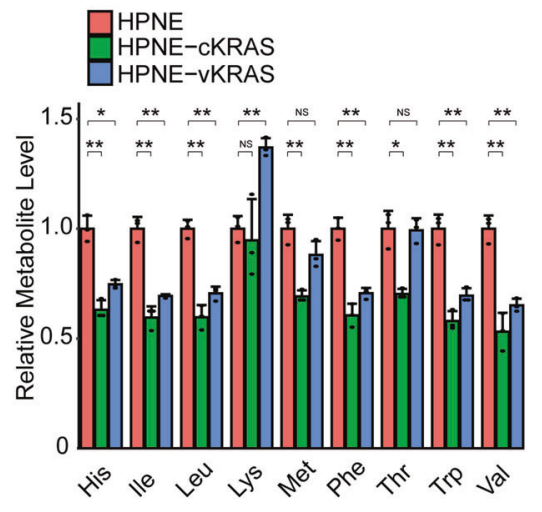

d

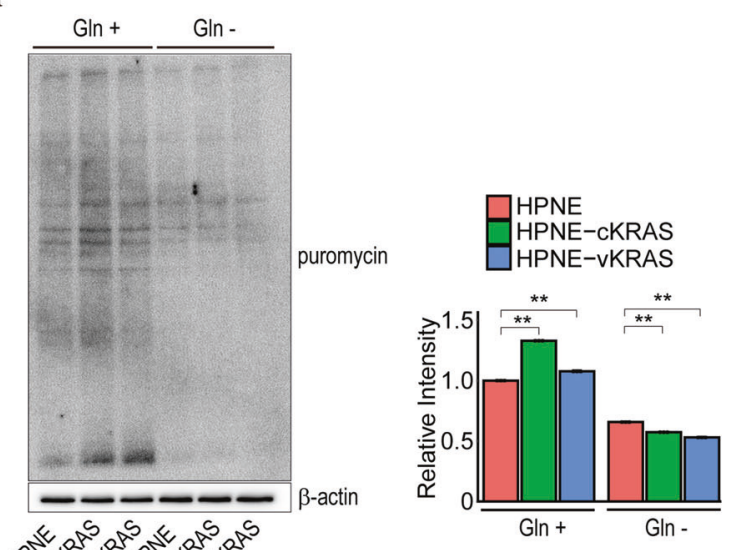

f

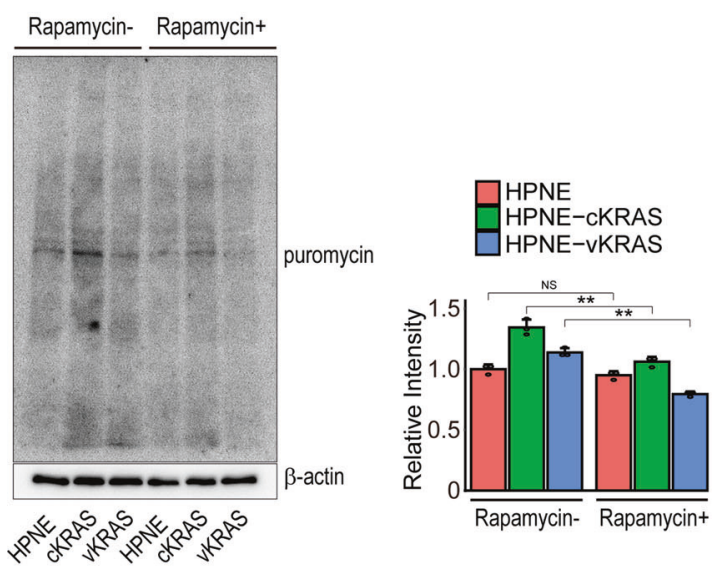

colon-vKRAS cells compared to control cells (Fig. 5a and Supplementary Fig. 6a). Accordingly, the protein synthesis in HPNE-cKRAS and HPNE-vKRAS cells (Fig. 5b) and the proliferation of HPNE-cKRAS, HPNE-vKRAS, and colon-vKRAS cells (Fig. 5c and Supplementary Fig. 6b) were significantly suppressed by the autophagy inhibitor chloroquine, suggesting that autophagy provides essential nutrient resources for the viability of KRAS mutant cells. To investigate further mechanisms of autophagy activation in KRAS mutant cells, we evaluated the autophagic flux with MEK inhibitor treatment. Consistent with previous reports with PDAC cells [46, 47], the inhibition of MEK, a downstream molecule of KRAS, activates autophagic flux regardless of KRAS state 
Fig. 4 Intracellular amino acid levels are decreased in KRAS mutant pancreatic epithelial cells. a Intracellular non-essential amino acid levels. Results are the means of three biological replicates; error bars represent SD. NS, not significant; $* p<0.05$; $* * p<0.01$. b Intracellular essential amino acid levels. Results are the means of three biological replicates; error bars represent SD. NS, not significant; ${ }^{*} p<0.05 ; * * p<0.01$. c Relative mRNA levels of the indicated genes taking the levels in HPNE cells as 1. Results are the means of three biological replicates; error bars represent SD. NS, not significant; $* p<0.05$; $* * p<0.01$. d Puromycin incorporation assay results. Cells were cultured in normal $(\mathrm{Gln}+)$ or glutamine-deficient (Gln-) medium for $24 \mathrm{~h}$. Puromycin was added 10 min before sample collection and the lysates were subjected to Western blotting using an anti-puromycin antibody. Representative results from three independent experiments are shown. Relative total band intensities are shown at the right. Error bars represent $\mathrm{SD}$. ${ }^{*} p<0.01$. e mTOR pathway activity as determined by measuring the phosphorylation level of p70S6K and 4E-BP1 by Western blotting. Representative results from three independent experiments are shown. Band intensities are indicated below the images. $\mathbf{f}$ Puromycin incorporation assay results. Cells were treated with or without $100 \mathrm{nM}$ rapamycin for $6 \mathrm{~h}$. Puromycin was added $10 \mathrm{~min}$ before sample collection and the lysates were subjected to Western blotting using an anti-puromycin antibody. Representative results from three independent experiments are shown. Relative total band intensities are shown at the right. Error bars represent the SD. $* * p<0.01$. NS, not significant; $* * p<0.01$.

(Supplementary Fig. 7), which indicated that other downstream pathways could regulate autophagic flux in HPNE-cKRAS and HPNE-vKRAS cells.

These findings imply that nutritional interventions with autophagy inhibition in non-cancerous epithelial cells can suppress or delay the development of PDAC.

\section{Discussion}

$K R A S$ mutation increased the dependency on glucose and glutamine for energy production and maintenance of the redox balance in human non-cancerous pancreatic cells. In addition, the intracellular amino acid levels were significantly decreased. Accordingly, the cells required greater autophagic flux for proliferation, as did tumor maintenance in PDAC mouse models [30, 48, 49]. Therefore, nutritional interventions and autophagy inhibition may prevent the proliferation of non-cancerous pancreatic cells with $K R A S$ mutation.

Evidence of the existence of metabolic reprogramming in KRAS-mutated PDAC cells has accumulated [18-23]. Our results also indicated that pancreatic non-cancerous cells with induced KRAS mutation are dependent on glucose and glutamine. Because pancreatic carcinogenesis is accompanied by KRAS mutation as an initial molecular event [10-12], these results suggest that metabolic reprogramming is induced by KRAS mutation at an early stage of pancreatic carcinogenesis.
Non-cancerous pancreatic cells with KRAS mutation exhibited markedly decreased intracellular amino acid levels. The mTOR pathway was upregulated in cells with KRAS mutation, indicating enhanced protein synthesis and amino acid consumption [44]. In addition, several amino acid transporters were highly expressed to meet the increased demand for amino acids in cells with KRAS mutation, as reported in PDAC [50, 51]. Among amino acids, asparagine and proline were markedly decreased in non-cancerous pancreatic cells with KRAS mutation. Because intracellular asparagine is exchanged for extracellular amino acids to regulate protein synthesis [52], asparagine may be consumed not only for protein synthesis but also to enhance the importation of other amino acids. In addition, because PDAC cells maintain their growth by producing proline as a substrate for the TCA cycle [53], the reduced proline levels in pancreatic cells with KRAS mutation are likely to reflect increased consumption of proline in the TCA cycle. Therefore, pancreatic cells with KRAS mutation may have extensive reprogramming of amino acid metabolism to maintain their viability.

Autophagic flux was increased in cells with KRAS mutation in this study, consistent with studies of PDAC [28, 48]. However, it remains to be clarified how KRAS drives the high basal levels of autophagy. To identify the relationship between KRAS mutation, KRAS activation, and autophagy, we examined the autophagic flux after treatment with an inhibitor of MEK, which is downstream of KRAS. MEK inhibition elevated rather than decreased the autophagic flux, as reported in PDAC [46, 47]. Although this may be a consequence of disrupted glycolysis and mitochondrial function by MEK inhibition, as discussed in PDAC, further study is needed to identify the direct and indirect regulators that lead to the compensatory increased autophagic flux induced by KRAS mutation.

$K R A S$ mutation is crucial for carcinogenesis in various tissues, not only the pancreas. Human colon polyclonal epithelial cells with KRAS mutation exhibited increased autophagic flux and were sensitive to autophagy inhibition, similar to pancreatic polyclonal epithelial cells with KRAS mutation. However, their phenotype was slightly different from those of pancreatic cells, in terms of, for example, PI3K pathway activity and ASNS expression, as reported previously [54]. Therefore, the KRAS mutation is universally crucial for carcinogenesis, its biological effects may be tissue or cell-type specific, as reported [55].

Gene-editing technology enables the examination of the effects of mutations. We compared the physiological effect of KRAS mutation using mutant knocked-in HPNE cells and $K R A S$ gene-overexpressing polyclonal cells. Although the phenotypes were mostly similar, several quantitative assays, such as protein translation levels and sensitivities to 
$\mathbf{a}$
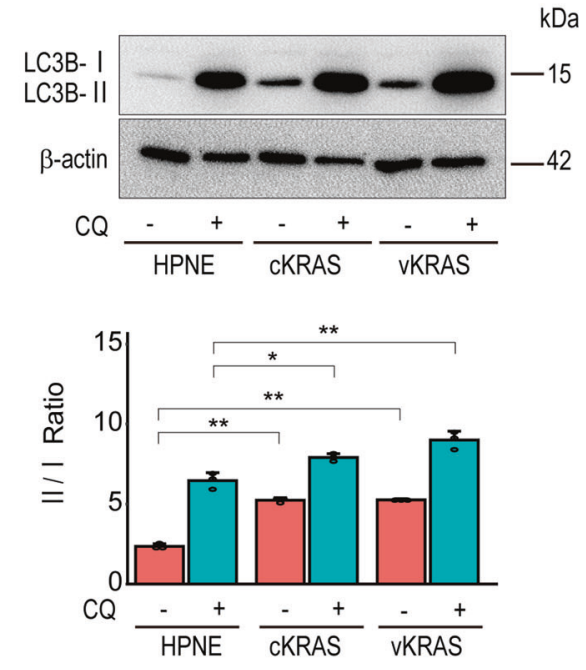

c

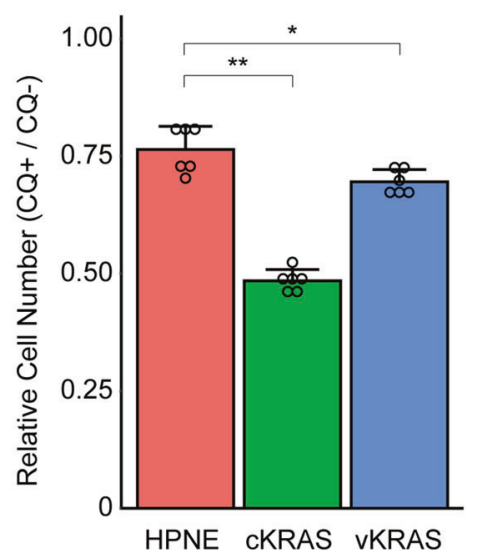

Fig. 5 Autophagy is required for maintaining $K R A S$ mutant pancreatic epithelial cells. a Autophagic flux levels as determined by Western blotting of LC3B-I and LC3B-II. Representative results from three independent experiments are shown. Band intensity ratios (LC3B-II/LC3B-I ratio) are indicated below the images. A lysosomal inhibitor, chloroquine (CQ), was used to assess autophagic flux by assessing the LC3B-II level. Error bars represent the SD. ${ }^{*} p<0.05$; $* * p<0.01$. b Puromycin incorporation assay results. Cells were

autophagy inhibition, gave different results. This is likely to be caused by differences in the mutant KRAS expression levels. Indeed, the mutant KRAS allelic content reportedly defines the degree of metabolic reprogramming [56] and KRAS gene amplification occurs during pancreatic carcinogenesis [57]. Therefore, mutation knock-in cells are more physiological and suitable for investigations of the early steps of carcinogenesis. Further, the effects of the lentiviral backbone used to generate the mutant KRAS geneoverexpressing cells cannot be ignored. Therefore, caution is needed when interpreting the results and performing functional analysis of mutant gene-overexpressing cells. b

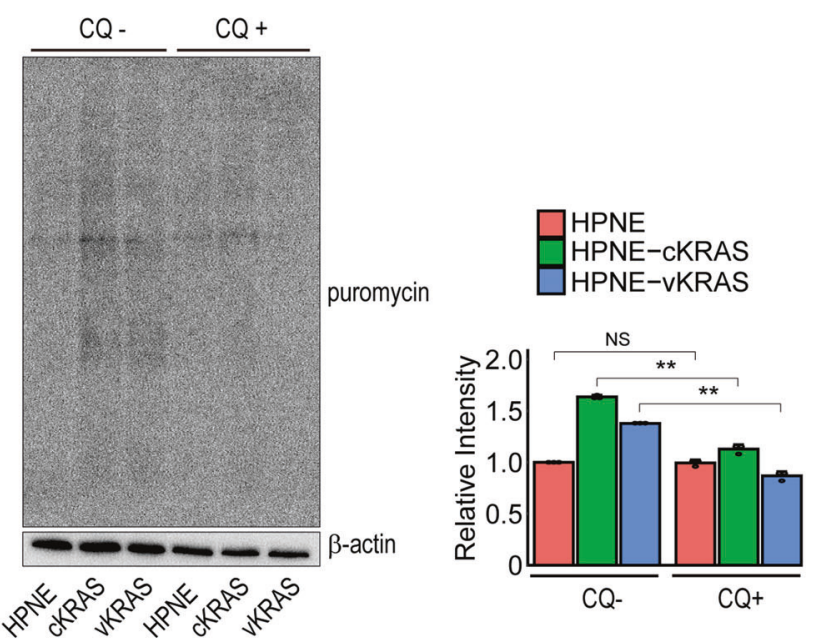

treated with or without $5 \mu \mathrm{M}$ chloroquine for $4 \mathrm{~h}$. Puromycin was added $10 \mathrm{~min}$ before sample collection. Representative results from three independent experiments are shown. Relative total band intensities are shown at the right. Error bars represent the SD. NS, not significant; $* * p<0.01$. c Cells were treated with $5 \mu \mathrm{M}$ chloroquine for $48 \mathrm{~h}$. The number of cells relative to the control (without treatment) was determined. Results are the means of six biological replicates; error bars represent the SD. $* p<0.05 ; * * p<0.01$.

In conclusion, KRAS mutation in human pancreatic ductal epithelial cells triggered diverse metabolic reprogramming and compensatory autophagy, which may create a favorable environment for cell proliferation and accelerate the progression to carcinogenesis. Although the pancreatic epithelial cells used in this study were immortalized, the results may provide us with the biological effects in the early phase of carcinogenesis, initialized by KRAS mutation in humans. Because the existence of cells with KRAS mutation in the body has recently been detected from plasma using cell-free DNA with high sensitivity [58], nutritional intervention including autophagy inhibition from 
the early stages of carcinogenesis may become an option to suppress or delay subsequent carcinogenesis in the future.

\section{Methods}

\section{Cell lines}

Human normal pancreatic epithelial (HPNE) cells immortalized with hTERT and human pancreatic carcinoma (CFPAC-1) cells carrying heterozygous $K R A S^{\mathrm{G} 12 \mathrm{~V}}$ mutation with KRAS copy number gain [59, 60] were obtained from the American Type Culture Collection (Manassas, VA). 293TN human embryonic kidney cells were purchased from System Biosciences (SBI, Mountain View, CA). Human normal colonic epithelial cells immortalized with SV40 large $\mathrm{T}$ antigen were purchased from Applied Biological Materials (Applied Biological Materials, Richmond, BC, Canada). HPNE cells and 293TN cells were cultured in Dulbecco's Modified Eagle's Medium (DMEM) (SigmaAldrich, St. Louis, MO, \#D6046) supplemented with 10\% fetal bovine serum (FBS). CFPAC-1 cells were cultured in Iscove's Modified Dulbecco's Medium (IMDM) (SigmaAldrich, \#I3390) supplemented with 10\% FBS. Human colonic epithelial cells were cultured in DMEM supplemented with $5 \%$ FBS. All cells were incubated at $37^{\circ} \mathrm{C}$ in an atmosphere containing $20 \% \mathrm{O}_{2}$ and $5 \% \mathrm{CO}_{2}$.

\section{Nutrient-deficient media}

Glucose-free and glutamine-free DMEM was purchased from Sigma-Aldrich (\#D5030) and supplemented with sodium bicarbonate (Wako Pure Chemical Industries, Osaka, Japan) and FBS. Immediately before use, glucose (Wako Pure Chemical Industries) and/or glutamine (Invitrogen, Carlsbad, CA) were added to 5.5 and $4 \mathrm{mM}$, respectively. For puromycin labeling assay and cellular ROS measurement, glutamine-free DMEM (Sigma-Aldrich, \#D5546) with FBS was used.

\section{Genome editing using the CRISPR/Cas9 system}

Cas9 protein, CRISPR RNA (crRNA) (KRAS \#1 and KRAS $\# 2$ ), and trans-crRNA (tracrRNA) were purchased from Integrated DNA Technologies (IDT, Coralville, IA). The crRNAs used were as follows: KRAS \#1, 5'-rCrUrGrAr ArUrUrArGrCrUrGrUrArUrCrGrUrCrArGrUrUrUrUrArGrAr GrCrUrArUrGrCrU-3' and KRAS \#2, 5'-rGrArArUrArUrAr ArArCrUrUrGrUrGrGrUrArGrUrGrUrUrUrUrArGrArGrCrUr ArUrGrCrU-3'. The single-stranded oligo DNAs (ssODNs) were purchased from Eurofins Genomics (Tokyo, Japan). The ssODNs used were as follows: ssODN for KRAS \#1, 5'TTTTCATTATTTTTAATTTTGTGGACGAAT-3'; ssODN for KRAS \#2, 5'-TACCTCTATTGTTGGATTTTCAGC AGGCCT-3'. HPNE cells were transfected according to the recommended protocol of IDT. To isolate cells with KRAS mutation, the mutation frequencies were examined by dropletdigital PCR (ddPCR) after repeated limiting dilution of the samples. Briefly, single-cell clones were seeded in a 96-well plate, cultured for several days, and genomic DNA was extracted from a randomly selected well using a DNA Extraction Kit (Kaneka, Tokyo, Japan). After nested PCR using primer pairs outside the ssODN sequences, ddPCR was performed using probes (Bio-Rad Laboratories Hercules, CA) for simultaneous detection of KRAS mutant allele (FAM)/wild allele (HEX) (\#10049550). CFPAC-1 cells were used as the positive control and HPNE cells as the negative control. Cell populations with high mutant frequencies were selected and seeded in 96-well plates with limiting dilution. The above procedure was repeated four times to concentrate the mutant allele to isolate cells heterozygous for a KRAS mutant allele. The primer sequences used are listed in Supplementary Table 1 .

\section{Transfection and lentivirus transduction}

For lentivirus-mediated KRAS ${ }^{\mathrm{G} 12 \mathrm{~V}}$ overexpression, pLentiPGK-KRAS4B (G12V) was obtained from Addgene (plasmid \#35633). The Lentivirus Packaging System (SBI) was used to generate stably expressing polyclonal cells according to the manufacturer's protocol. Briefly, the pLenti-PGKKRAS4B (G12V) plasmid and pPACKH1 packaging plasmid mix were transfected into 293TN cells using Effectene Transfection Reagent (QIAGEN, Hilden, Germany). After $48 \mathrm{~h}$, the culture media were collected and the viruses were concentrated using PEG-it Reagent (SBI). The centrifuged pellet was resuspended in $1 \times \mathrm{PBS}$, and aliquots were stored at $-80^{\circ} \mathrm{C}$. Viruses were added to the target cells with polybrene reagent (Santa Cruz Biotechnology, Dallas, TX), followed by selection with hygromycin.

\section{Cell growth assay}

The Cell Counting Kit-8 (Dojindo Molecular Technologies, Kumamoto, Japan) was used to assay cell growth according to the manufacturer's protocol. Briefly, $1.0 \times 10^{4}$ cells were seeded in 96 -well plates. Next, $10 \mu \mathrm{L}$ of CCK-8 solution were added, the plates were incubated for $60 \mathrm{~min}$ at $37^{\circ} \mathrm{C}$, and the absorbance at $450 \mathrm{~nm}$ was measured using a microplate reader (Thermo Fisher Scientific, Waltham, MA).

\section{RAS activity assay}

RAS activity was measured using the 96-well Ras Activation ELISA Kit (Cell Biolabs, San Diego, CA). Briefly, $4.0 \times 10^{5}$ cells were cultured on a 6 -well dish and lysate 
samples were extracted using Assay/Lysis Buffer. Then, the lysates were added to each well of the plate and the active form of RAS in the samples was selectively isolated with plate-bound Raf-1 RBD, followed by incubation with an anti-pan-Ras antibody and HRP-conjugated secondary antibody. The absorbance was measured at $450 \mathrm{~nm}$ by a microplate reader (Thermo Fisher Scientific).

\section{Western blotting and antibodies}

Western blotting was performed as described previously [61]. Briefly, lysate samples were separated in 10-20\% gradient polyacrylamide gels and transferred to polyvinylidene difluoride membranes (GE Healthcare, Little Chalfont, UK). After blocking with 5\% dry milk, the membranes were probed with the appropriate primary antibodies diluted in Immunoshot Reagent 1 (Cosmo bio, Tokyo, Japan). The corresponding horseradish peroxidase (HRP)-conjugated secondary antibodies (GE Healthcare) were subsequently added. Bound antibodies were detected using the Immunostar LD Reagents (Wako Pure Chemical Industries). Antibodies against the following were used: $\beta$-actin (\#5125, 1:10000), Ras (G12V Mutant Specific) (D2H12) (\#14412, 1:1000), Ras (\#3965, 1:1000), phosphop44/42 MAPK (Erk1/2) (Thr202/Tyr204) (\#9101, 1:1000), p44/42 MAPK (Erk1/2) (\#9102, 1:1000), phospho-Akt (Ser473) (D9E) (\#4060, 1:1000), Akt (\#9272, 1:1000), Hexokinase II (C64G5) (\#2867, 1:1000), LDHA (C4B5) (\#3582, 1:1000), phospho-p70 S6 kinase (Thr389) (\#9205, 1:1000), p70 S6 kinase (49D7) (\#2708, 1:1000), phospho4E-BP1 (Thr70) (\#9455, 1:1000), 4E-BP1 (53H11) (\#9644, 1:1000), phospho-AMPK $\alpha$ (Thr172) (D4D6D) (\#50081, 1:1000), AMPK $\alpha$ (D5A2) (\#5831, 1:1000), and LC3B (D11) (\#3868, 1:1000) (Cell Signaling Technology, Danvers, MA). GLUD1 (ab166618, 1:1000) (Abcam, Cambridge, MA). GOT1 (AV48205, 1:1000) (Sigma). Uncropped images are in Supplementary Figs. 8 and 9.

\section{Puromycin-labeling assay}

The puromycin-labeling assay was performed as described previously [43]. Briefly, cells were cultured in the presence and absence of glutamine for $24 \mathrm{~h}$. Puromycin $(90 \mathrm{mM})$ was added to the culture medium for $10 \mathrm{~min}$, and the cells were rigorously washed and harvested for Western blotting using an anti-puromycin antibody (MABE343, 1:1000; Merck Millipore, Burlington, MA).

\section{RNA extraction, reverse transcription, and quantitative real-time PCR}

Total RNA was isolated from $1.0 \times 10^{6}$ cells using ISOGEN II (Nippon Gene, Toyama, Japan) according to the manufacturer's protocol. Reverse transcription was performed using the SuperScript III First-Strand Synthesis System for RT-PCR (Invitrogen). Next, $100 \mathrm{ng}$ of template were subjected to quantitative SYBR Green PCR (StepOnePlus $^{\mathrm{TM}}$ Realtime PCR System; Invitrogen). All qPCR tests were run in triplicate. The average threshold cycle number $(\mathrm{Ct})$ values were normalized to that of actin and calculated using the delta-delta $\mathrm{Ct}(\mathrm{ddCt})$ method. The primer sequences used are listed in Supplementary Table 2.

\section{Glucose-uptake assay}

Glucose-uptake assay was performed as described previously [62]. Briefly, $4.0 \times 10^{5}$ cells were treated with $100 \mu \mathrm{M}$ fluorescently labeled deoxyglucose analog, 2-(N(7-nitrobenz-2-oxa-1,3-diazol-4-yl)amino)-2-deoxyglucose (2-NBDG; Invitrogen) [63] for $30 \mathrm{~min}$. The cells were trypsinized and single-cell suspensions were analyzed by flow cytometry using Guava Easy Cyte Plus (GE Healthcare). As a result of differences in endogenous fluorescence between cells, 2NBDG-mediated fluorescence intensities were normalized to those of unlabeled cells.

\section{Cellular lactate measurement}

The cellular lactate level was measured using the Lactate Assay Kit-WST (Dojindo Molecular Technologies) according to the manufacturer's protocol. Briefly, $1.0 \times 10^{4}$ cells were seeded in 96-well plates, and the plates were incubated overnight at $37^{\circ} \mathrm{C}$. The medium was exchanged for fresh medium, and the cells were incubated for $24 \mathrm{~h}$. Next, $20 \mu \mathrm{L}$ of culture supernatant was transferred to a 96well plate, $80 \mu \mathrm{L}$ of the working solution was added, and the plates were incubated for $30 \mathrm{~min}$ at $37^{\circ} \mathrm{C}$. Finally, the absorbance at $450 \mathrm{~nm}$ was measured using a microplate reader (Thermo Fisher Scientific).

\section{Measurement of oxygen consumption rate}

Oxygen consumption rate was measured using an Oxygen Consumption Rate Assay Kit (Cayman Chemical, Ann Arbor, MI) according to the manufacturer's protocol. Briefly, $2.0 \times 10^{4}$ pre-cultured cells in 96-well plates were incubated overnight at $37^{\circ} \mathrm{C}$. The medium was then replaced with $160 \mu \mathrm{L}$ of fresh medium, and a phosphorescent probe was added to measure the oxygen consumption. Each well was sealed with $100 \mu \mathrm{L}$ of mineral oil to prevent oxygen diffusion. The signals were measured by an ARVO X5 plate reader (PerkinElmer Japan, Kanagawa, Japan) using time-resolved mode at Ex $380 \mathrm{~nm}$ and $\mathrm{Em} 650 \mathrm{~nm}$ for $210 \mathrm{~min}$ at 1-min intervals. Linear regression was performed after subtracting the blank, and the oxygen consumption rate was indicated by the slope of each signal profile. 


\section{Assessment of mitochondrial DNA copy number}

Total DNA was isolated from $1.0 \times 10^{6}$ cells using the QIAamp DNA Mini Kit (QIAGEN) according to the manufacturer's protocol. Next, $100 \mathrm{ng}$ of template were subjected to qPCR. The average $\mathrm{Ct}$ values of mitochondrial and nuclear DNA were obtained. The relative content of mitochondrial DNA, normalized to that of nuclear DNA, was calculated using the ddCt method. As primers, Human Mitochondrial DNA (mtDNA) Monitoring Primer Sets (TaKaRa, Shiga, Japan) targeting ND1 and ND5 as mitochondrial DNAs and SLCO2B1 and SERPINA1 as nuclear DNAs were used.

\section{Assay of cellular ATP levels}

Cellular ATP levels were measured using the CellTiter-Glo Luminescent Cell Viability Assay Kit (Promega, Fitchburg, WI). Briefly, $1.0 \times 10^{4}$ cells were cultured in $100 \mu \mathrm{L}$ of the medium on 96 -well plates. Next, $100 \mu \mathrm{L}$ of CellTiter-Glo Reagent was added and mixed for 2 min on an orbital shaker to induce cell lysis. Subsequently, the plates were incubated for $10 \mathrm{~min}$ at RT, and the luminescence was measured using a GloMax 96 Microplate Luminometer (Promega).

\section{Assay of cellular ROS levels}

Cellular ROS levels were measured using the ROS-Glo $\mathrm{H}_{2} \mathrm{O}_{2}$ Assay Kit (Promega). Briefly, $1.0 \times 10^{4}$ cells were cultured in $80 \mu \mathrm{L}$ of the medium on 96-well plates. Next, $20 \mu \mathrm{L}$ of the $\mathrm{H}_{2} \mathrm{O}_{2}$ substrate solution was added, followed by incubation for $6 \mathrm{~h}$ in the indicated medium. Subsequently, $100 \mu \mathrm{L}$ of ROS-Glo Detection Solution were added and the plates were incubated for $20 \mathrm{~min}$ at RT, followed by measurement of luminescence using a GloMax 96 Microplate Luminometer (Promega).

\section{Metabolome analysis}

Metabolome analysis was performed by Human Metabolome Technologies (HMT, Tsuruoka, Japan), according to their methods. Briefly, $1.0 \times 10^{6}$ cells were seeded onto $10 \mathrm{~cm}$ dishes for $24 \mathrm{~h}$, and metabolites were extracted. The cells were washed twice with $5 \%$ mannitol and treated with $800 \mathrm{~mL}$ of methanol and $550 \mathrm{~mL}$ of water containing internal standards (HMT). The extract was filtered using an UltrafreeMCPLHCC $5 \mathrm{kDa}$-cutoff filter unit (HMT) by centrifugation at $9100 \times g$, at $4{ }^{\circ} \mathrm{C}$ for $120 \mathrm{~min}$. Metabolite extracts were prepared as biological triplicates for each sample. Cationic compounds were measured in the positive mode of CE-TOFMS, and anionic compounds were measured in the positive and negative modes of CE-MS/MS as described previously [64].
The peaks detected by CE-TOFMS and CE-MS/MS were extracted using automatic integration software [65] (MasterHands, Keio University, Tsuruoka, Japan) and MassHunter Quantitative Analysis B.04.00, Agilent Technologies, Santa Clara, CA) to obtain peak information, including $\mathrm{m} / \mathrm{z}$, migration time (MT), and peak area. The peaks were annotated with putative metabolites from the HMT metabolite database based on their MTs in CE and $\mathrm{m} / \mathrm{z}$ values determined by TOFMS and MS/MS. The tolerance range for the peak annotation was set to $\pm 0.5 \mathrm{~min}$ for MT and $\pm 10 \mathrm{ppm}$ for $\mathrm{m} / \mathrm{z}$. Metabolite concentrations were calculated by normalizing the peak area of each metabolite to that of the internal standard and by using standard curves generated from three-point calibrations.

\section{Analysis of autophagic flux}

Cells were treated with chloroquine $(5 \mu \mathrm{M})$ for $2 \mathrm{~h}$. Autophagic flux was quantified by determining the ratio of the band intensities of LC3II to LC3BI after Western blotting.

\section{Statistical analysis}

The statistical significance of differences between groups was evaluated by Student's $t$-test when the variances were equal. When the variances were unequal, Welch's $t$-test was used instead. $P$-values less than 0.05 were considered to indicate statistical significance.

\section{Data availability}

The authors declare that all the data supporting the findings of this study are available within the article and its supplementary information files and from the corresponding author upon reasonable request.

Acknowledgements We thank Ms. Sayaka Ito for her technical assistance. This work was supported by Grants-in-Aid from the Ministry of Education, Culture, Sports, Science and Technology, Japan (\#19H03430, \#20J11303, \#19J11829, and \#19K23881) (to M.Otsuka, T.Seimiya, K.S., and R.I.), by JST CREST (to M.Otsuka, \#JPMJCR19H5), and by AMED (to M.Otsuka, \#JP20ck0106557).

Author contributions T.Suzuki and M.Otsuka designed the research. T.Suzuki, T.K., T.Seimiya., K.S., M.Ohno, T.I., and R.I. performed the majority of the experiments. T.Suzuki, T.Sato, N.T., and Y.S. analyzed the data. T.Suzuki, M.Otsuka, and K.K. wrote the manuscript.

\section{Compliance with ethical standards}

Conflict of interest The authors declare no competing interests.

Publisher's note Springer Nature remains neutral with regard to jurisdictional claims in published maps and institutional affiliations. 
Open Access This article is licensed under a Creative Commons Attribution 4.0 International License, which permits use, sharing, adaptation, distribution and reproduction in any medium or format, as long as you give appropriate credit to the original author(s) and the source, provide a link to the Creative Commons license, and indicate if changes were made. The images or other third party material in this article are included in the article's Creative Commons license, unless indicated otherwise in a credit line to the material. If material is not included in the article's Creative Commons license and your intended use is not permitted by statutory regulation or exceeds the permitted use, you will need to obtain permission directly from the copyright holder. To view a copy of this license, visit http://creativecommons. org/licenses/by/4.0/.

\section{References}

1. Siegel RL, Miller KD, Jemal A. Cancer statistics, 2019. CA 2019;69:7-34.

2. Kleeff J, Korc M, Apte M, La Vecchia C, Johnson CD, Biankin AV, et al. Pancreatic cancer. Nat Rev Dis Prim. 2016;2:16022.

3. Janes MR, Zhang J, Li LS, Hansen R, Peters U, Guo X, et al. Targeting KRAS mutant cancers with a covalent G12C-specific inhibitor. Cell. 2018;172:578-89.e517.

4. Jones S, Zhang X, Parsons DW, Lin JC, Leary RJ, Angenendt P, et al. Core signaling pathways in human pancreatic cancers revealed by global genomic analyses. Science. 2008;321:1801-6.

5. Bailey P, Chang DK, Nones K, Johns AL, Patch AM, Gingras $\mathrm{MC}$, et al. Genomic analyses identify molecular subtypes of pancreatic cancer. Nature. 2016;531:47-52.

6. Waddell N, Pajic M, Patch AM, Chang DK, Kassahn KS, Bailey $\mathrm{P}$, et al. Whole genomes redefine the mutational landscape of pancreatic cancer. Nature. 2015;518:495-501.

7. Raphael BJ, Hruban RH, Aguirre AJ, Moffitt RA, Yeh JJ, Stewart $\mathrm{C}$, et al. Integrated genomic characterization of pancreatic ductal adenocarcinoma. Cancer Cell. 2017;32:185-203.

8. Connor AA, Denroche RE, Jang GH, Lemire M, Zhang A, ChanSeng-Yue $M$, et al. Integration of genomic and transcriptional features in pancreatic cancer reveals increased cell cycle progression in metastases. Cancer Cell. 2019;35:267-82.e267.

9. Papke B, Der CJ, Drugging RAS. Know the enemy. Science. 2017;355:1158-63.

10. Almoguera C, Shibata D, Forrester K, Martin J, Arnheim N, Perucho M. Most human carcinomas of the exocrine pancreas contain mutant C-K-Ras genes. Cell. 1988;53:549-54.

11. Hruban RH, Goggins M, Parsons J, Kern SE. Progression model for pancreatic cancer. Clin Cancer Res. 2000;6:2969-72.

12. Hingorani SR, Petricoin EF, Maitra A, Rajapakse V, King C, Jacobetz MA, et al. Preinvasive and invasive ductal pancreatic cancer and its early detection in the mouse. Cancer Cell. 2003;4:437-50.

13. Pylayeva-Gupta Y, Grabocka E, Bar-Sagi D. RAS oncogenes: weaving a tumorigenic web. Nat Rev Cancer. 2011;11:761-74.

14. Lito P, Solomon M, Li LS, Hansen R, Rosen N. Allele-specific inhibitors inactivate mutant KRAS G12C by a trapping mechanism. Science. 2016;351:604-8.

15. Simanshu DK, Nissley DV, McCormick F. RAS proteins and their regulators in human disease. Cell. 2017;170:17-33.

16. Xue JY, Zhao Y, Aronowitz J, Mai TT, Vides A, Qeriqi B, et al. Rapid non-uniform adaptation to conformation-specific KRAS (G12C) inhibition. Nature. 2020;577:421-5.

17. Downward J, Targeting RAS. signalling pathways in cancer therapy. Nat Rev Cancer. 2003;3:11-22.

18. Ying H, Kimmelman AC, Lyssiotis CA, Hua S, Chu GC, Fletcher-Sananikone E, et al. Oncogenic Kras maintains pancreatic tumors through regulation of anabolic glucose metabolism. Cell. 2012;149:656-70.
19. Amendola CR, Mahaffey JP, Parker SJ, Ahearn IM, Chen W-C, Zhou M, et al. KRAS4A directly regulates hexokinase 1 . Nature. 2019;576:482-6.

20. Santana-Codina N, Roeth AA, Zhang Y, Yang A, Mashadova O, Asara JM, et al. Oncogenic KRAS supports pancreatic cancer through regulation of nucleotide synthesis. Nat Commun. 2018;9:4945.

21. Commisso C, Davidson SM, Soydaner-Azeloglu RG, Parker SJ, Kamphorst JJ, Hackett S, et al. Macropinocytosis of protein is an amino acid supply route in Ras-transformed cells. Nature. 2013;497:633-7.

22. Ramirez C, Hauser AD, Vucic EA, Bar-Sagi D. Plasma membrane V-ATPase controls oncogenic RAS-induced macropinocytosis. Nature. 2019;576:477-81.

23. Yao W, Rose JL, Wang W, Seth S, Jiang H, Taguchi A, et al. Syndecan 1 is a critical mediator of macropinocytosis in pancreatic cancer. Nature. 2019;568:410-4.

24. Halbrook CJ, Lyssiotis CA. Employing metabolism to improve the diagnosis and treatment of pancreatic cancer. Cancer Cell. 2017;31:5-19.

25. McDonald PC, Chafe SC, Brown WS, Saberi S, Swayampakula $\mathrm{M}$, Venkateswaran $\mathrm{G}$, et al. Regulation of $\mathrm{pH}$ by carbonic anhydrase 9 mediates survival of pancreatic cancer cells with activated KRAS in response to hypoxia. Gastroenterology. 2019;157:823-37.

26. Son J, Lyssiotis CA, Ying H, Wang X, Hua S, Ligorio M, et al. Glutamine supports pancreatic cancer growth through a KRASregulated metabolic pathway. Nature. 2013;496:101-5.

27. Suzuki T, Otsuka M, Seimiya T, Iwata T, Kishikawa T, Koike K. The biological role of metabolic reprogramming in pancreatic cancer. MedComm. 2020;1:302-10.

28. Yang S, Wang X, Contino G, Liesa M, Sahin E, Ying H, et al. Pancreatic cancers require autophagy for tumor growth. Genes Dev. 2011;25:717-29.

29. Sousa CM, Biancur DE, Wang X, Halbrook CJ, Sherman MH, Zhang L, et al. Pancreatic stellate cells support tumour metabolism through autophagic alanine secretion. Nature. 2016;536:479-83.

30. Yang A, Herter-Sprie G, Zhang H, Lin EY, Biancur D, Wang X, et al. Autophagy sustains pancreatic cancer growth through both cell-autonomous and nonautonomous mechanisms. Cancer Discov. 2018;8:276-87.

31. Galluzzi L, Pietrocola F, Levine B, Kroemer G. Metabolic control of autophagy. Cell. 2014;159:1263-76.

32. Mizushima N, Komatsu M. Autophagy: renovation of cells and tissues. Cell. 2011;147:728-41.

33. Liou GY, Doppler H, DelGiorno KE, Zhang L, Leitges M, Crawford HC, et al. Mutant KRas-induced mitochondrial oxidative stress in acinar cells upregulates EGFR signaling to drive formation of pancreatic precancerous lesions. Cell Rep. 2016; 14:2325-36.

34. Carrer A, Trefely S, Zhao S, Campbell SL, Norgard RJ, Schultz $\mathrm{KC}$, et al. Acetyl-CoA metabolism supports multistep pancreatic tumorigenesis. Cancer Discov. 2019;9:416-35.

35. Levine AJ, Jenkins NA, Copeland NG. The roles of initiating truncal mutations in human cancers: the order of mutations and tumor cell type matters. Cancer Cell. 2019;35:10-15.

36. Findlay SD, Vincent KM, Berman JR, Postovit LM. A digital PCR-based method for efficient and highly specific screening of genome edited cells. PLoS ONE. 2016;11:e0153901.

37. Chang Z, Ju H, Ling J, Zhuang Z, Li Z, Wang H, et al. Cooperativity of oncogenic K-ras and downregulated p16/INK4A in human pancreatic tumorigenesis. PLoS ONE. 2014;9:e101452.

38. Altman BJ, Stine ZE, Dang CV. From Krebs to clinic: glutamine metabolism to cancer therapy. Nat Rev Cancer. 2016;16:619-34.

39. Fan J, Kamphorst JJ, Mathew R, Chung MK, White E, Shlomi T, et al. Glutamine-driven oxidative phosphorylation is a major ATP 
source in transformed mammalian cells in both normoxia and hypoxia. Mol Syst Biol. 2013;9:712.

40. Reznik E, Miller ML, Senbabaoglu Y, Riaz N, Sarungbam J, Tickoo SK, et al. Mitochondrial DNA copy number variation across human cancers. eLife 2016;5:e10769.

41. Shadel GS, Horvath TL. Mitochondrial ROS signaling in organismal homeostasis. Cell. 2015;163:560-9.

42. Giustarini D, Colombo G, Garavaglia ML, Astori E, Portinaro NM, Reggiani F, et al. Assessment of glutathione/glutathione disulphide ratio and S-glutathionylated proteins in human blood, solid tissues, and cultured cells. Free Radic Biol Med. 2017;112:360-75.

43. Pavlova NN, Hui S, Ghergurovich JM, Fan J, Intlekofer AM, White RM, et al. As extracellular glutamine levels decline, asparagine becomes an essential amino acid. Cell Metab. 2018;27:428-38.

44. Nicklin P, Bergman P, Zhang B, Triantafellow E, Wang H, Nyfeler B, et al. Bidirectional transport of amino acids regulates mTOR and autophagy. Cell. 2009;136:521-34.

45. Knott SRV, Wagenblast E, Khan S, Kim SY, Soto M, Wagner M, et al. Asparagine bioavailability governs metastasis in a model of breast cancer. Nature. 2018;554:378-81.

46. Bryant KL, Stalnecker CA, Zeitouni D, Klomp JE, Peng S, Tikunov AP, et al. Combination of ERK and autophagy inhibition as a treatment approach for pancreatic cancer. Nat Med. 2019;25:628-40.

47. Kinsey CG, Camolotto SA, Boespflug AM, Guillen KP, Foth M, Truong A, et al. Protective autophagy elicited by RAF->MEK$>$ ERK inhibition suggests a treatment strategy for RAS-driven cancers. Nat Med. 2019;25:620-7.

48. Yang A, Rajeshkumar NV, Wang X, Yabuuchi S, Alexander BM, Chu GC, et al. Autophagy is critical for pancreatic tumor growth and progression in tumors with p53 alterations. Cancer Discov. 2014;4:905-13.

49. Rosenfeldt MT, O'Prey J, Morton JP, Nixon C, MacKay G, Mrowinska A, et al. p53 status determines the role of autophagy in pancreatic tumour development. Nature. 2013;504:296-300.

50. Coothankandaswamy V, Cao S, Xu Y, Prasad PD, Singh PK, Reynolds CP, et al. Amino acid transporter SLC6A14 is a novel and effective drug target for pancreatic cancer. $\mathrm{Br} \mathrm{J}$ Pharm. 2016;173:3292-306.

51. Kaira K, Sunose Y, Arakawa K, Ogawa T, Sunaga N, Shimizu K, et al. Prognostic significance of L-type amino-acid transporter 1 expression in surgically resected pancreatic cancer. Br J Cancer. 2012;107:632-8.

52. Krall AS, Xu S, Graeber TG, Braas D, Christofk HR. Asparagine promotes cancer cell proliferation through use as an amino acid exchange factor. Nat Commun. 2016;7:11457.
53. Olivares O, Mayers JR, Gouirand V, Torrence ME, Gicquel T, Borge L, et al. Collagen-derived proline promotes pancreatic ductal adenocarcinoma cell survival under nutrient limited conditions. Nat Commun. 2017;8:16031.

54. Toda K, Kawada K, Iwamoto M, Inamoto S, Sasazuki T, Shirasawa S, et al. Metabolic alterations caused by KRAS mutations in colorectal cancer contribute to cell adaptation to glutamine depletion by upregulation of asparagine synthetase. Neoplasia. 2016;18:654-65.

55. Yuneva MO, Fan TW, Allen TD, Higashi RM, Ferraris DV, Tsukamoto $\mathrm{T}$, et al. The metabolic profile of tumors depends on both the responsible genetic lesion and tissue type. Cell Metab. 2012;15:157-70.

56. Kerr EM, Gaude E, Turrell FK, Frezza C, Martins CP. Mutant Kras copy number defines metabolic reprogramming and therapeutic susceptibilities. Nature. 2016;531:110-3.

57. Mueller S, Engleitner T, Maresch R, Zukowska M, Lange S, Kaltenbacher T, et al. Evolutionary routes and KRAS dosage define pancreatic cancer phenotypes. Nature. 2018;554:62-8.

58. Mohan S, Ayub M, Rothwell DG, Gulati S, Kilerci B, Hollebecque A, et al. Analysis of circulating cell-free DNA identifies KRAS copy number gain and mutation as a novel prognostic marker in Pancreatic cancer. Sci Rep. 2019;9:11610.

59. Schoumacher RA, Ram J, Iannuzzi MC, Bradbury NA, Wallace RW, Hon CT, et al. A cystic fibrosis pancreatic adenocarcinoma cell line. Proc Natl Acad Sci USA. 1990;87:4012-6.

60. Hamidi H, Lu M, Chau K, Anderson L, Fejzo M, Ginther C, et al. KRAS mutational subtype and copy number predict in vitro response of human pancreatic cancer cell lines to MEK inhibition. Br J Cancer. 2014;111:1788-801.

61. Yoshikawa T, Wu J, Otsuka M, Kishikawa T, Suzuki N, Takata A, et al. Repression of MicroRNA function mediates inflammation-associated colon tumorigenesis. Gastroenterology. 2017;152:631-43.

62. Kottakis F, Nicolay BN, Roumane A, Karnik R, Gu H, Nagle JM, et al. LKB1 loss links serine metabolism to DNA methylation and tumorigenesis. Nature. 2016;539:390-5.

63. Yoshioka K, Takahashi H, Homma T, Saito M, Oh KB, Nemoto $\mathrm{Y}$, et al. A novel fluorescent derivative of glucose applicable to the assessment of glucose uptake activity of Escherichia coli. Biochim Biophys Acta. 1996;1289:5-9.

64. Soga T, Heiger DN. Amino acid analysis by capillary electrophoresis electrospray ionization mass spectrometry. Anal Chem. 2000;72:1236-41.

65. Sugimoto M, Wong DT, Hirayama A, Soga T, Tomita M. Capillary electrophoresis mass spectrometry-based saliva metabolomics identified oral, breast and pancreatic cancer-specific profiles. Metabolomics. 2010;6:78-95. 